\title{
Identification and characterization of the Cucurbitacins, a novel class of small- molecule inhibitors of Tropomyosin receptor kinase a
}

Yueling Zhong ${ }^{1 \dagger}$, Hong Xu ${ }^{2 \dagger}$, Yi Zhong ${ }^{1 \dagger}$, Xuemiao Zhang ${ }^{2}$, Ting Zeng ${ }^{3}$, Limei Li ${ }^{1}$, Gaojie Xu' ${ }^{1}$ Minhui Li ${ }^{3}$, Jin Liu ${ }^{1,3^{*}}$ and Tai Yang ${ }^{1,3^{*}}$ (D)

\begin{abstract}
Background: NGF-TrkA is well known to play a key role in propagating and sustaining pruritogenic signals, which form the pathology of chronic pruritus. Inhibition of NGF-TrkA is a known strategy for the treatment of pruritus. In the present paper, we describe the identification, in vitro characterization, structure-activity analysis, and inhibitory evaluation of a novel TrkA inhibitory scaffold exemplified by Cucurbitacins (Cus).

Methods: Cus were identified as TrkA inhibitors in a large-scale kinase library screen. To obtain structural models of Cus as TrkA inhibitors, AutoDock was used to explore their binding to TrkA. Furthermore, PC12 cell culture systems have been used to study the effects of Cus and traditional Chinese medicinal plants (Tian Gua Di and bitter gourd leaf) extracts on the kinase activity of TrkA.

Results: Cus block the phosphorylation of TrkA on several tyrosine sites, including Tyr490, Tyr674/675, and Tyr785, and inhibit downstream Akt and MAPK phosphorylation in response to NGF in PC12 cell model systems.

Furthermore, traditional Chinese medicinal plants (Tian Gua Di and bitter gourd leaf) containing Cu extracts were shown to inhibit the phosphorylation of TrkA and Akt. These data reveal mechanisms, at least partly, of the antipruritus bioactivity of Cus.
\end{abstract}

Conclusion: Taken together, with the recent discovery of the important role of TrkA as a therapeutic target, Cus could be the basis for the design of improved TrkA kinase inhibitors, which could someday help treat pruritus.

Keywords: Cucurbitacins, TrkA, Kinase, Inhibitors, Pruritus

\section{Background}

Cucurbitacins (Cus) are tetracyclic triterpenes, consisting of at least five cucurbitacin compounds, named Cucurbitacin B (CuB), Cucurbitacin D (CuD), Cucurbitacin E (CuE), Cucurbitacin I (CuI), and Cucurbitacin Q (CuQ). Among them, $\mathrm{CuB}, \mathrm{CuE}$, and $\mathrm{CuI}$ (Fig. 1a) have been widely studied. They are widely distributed in the Cucurbitaceae family and several other plants families, and have a range of biological and pharmacological activities. Cus were first

\footnotetext{
* Correspondence: jinliu@cmc.edu.cn; taiyang@cmc.edu.cn

†Yueling Zhong, Hong Xu and Yi Zhong contributed equally to this work. ${ }^{1}$ School of Pharmacy, Chengdu Medical College, No.783, Xindu Avenue, Xindu District, Chengdu 610500, Chengdu Sichuan Province, China Full list of author information is available at the end of the article
}

used as an anti-hepatitis drug in the late 1970s in China [1-3]. Interest in Cus has grown in recent years, and various studies have demonstrated that $\mathrm{Cu}$ analogues have a wide range of biological activities, including, hepatoprotective, anti-cancer, and anti-inflammatory activities [4]. However, biological targets of the Cus or detailed molecular mechanisms underlying their biological activities remain elusive. Some studies have demonstrated that their antiproliferative effects are associated with cell cycle arrest and apoptosis, mediated via inhibition of the Jak-STAT3 signaling pathway $[5,6]$, while others have indicated that Cus target cofilin, a critical mediator of actin dynamics, thereby disrupting actin assembly [7-9]. Our previous study showed that Cucurbitacin B (CuB)

(c) The Author(s). 2019 Open Access This article is distributed under the terms of the Creative Commons Attribution 4.0 International License (http://creativecommons.org/licenses/by/4.0/), which permits unrestricted use, distribution, and reproduction in any medium, provided you give appropriate credit to the original author(s) and the source, provide a link to the Creative Commons license, and indicate if changes were made. The Creative Commons Public Domain Dedication waiver (http://creativecommons.org/publicdomain/zero/1.0/) applies to the data made available in this article, unless otherwise stated. 

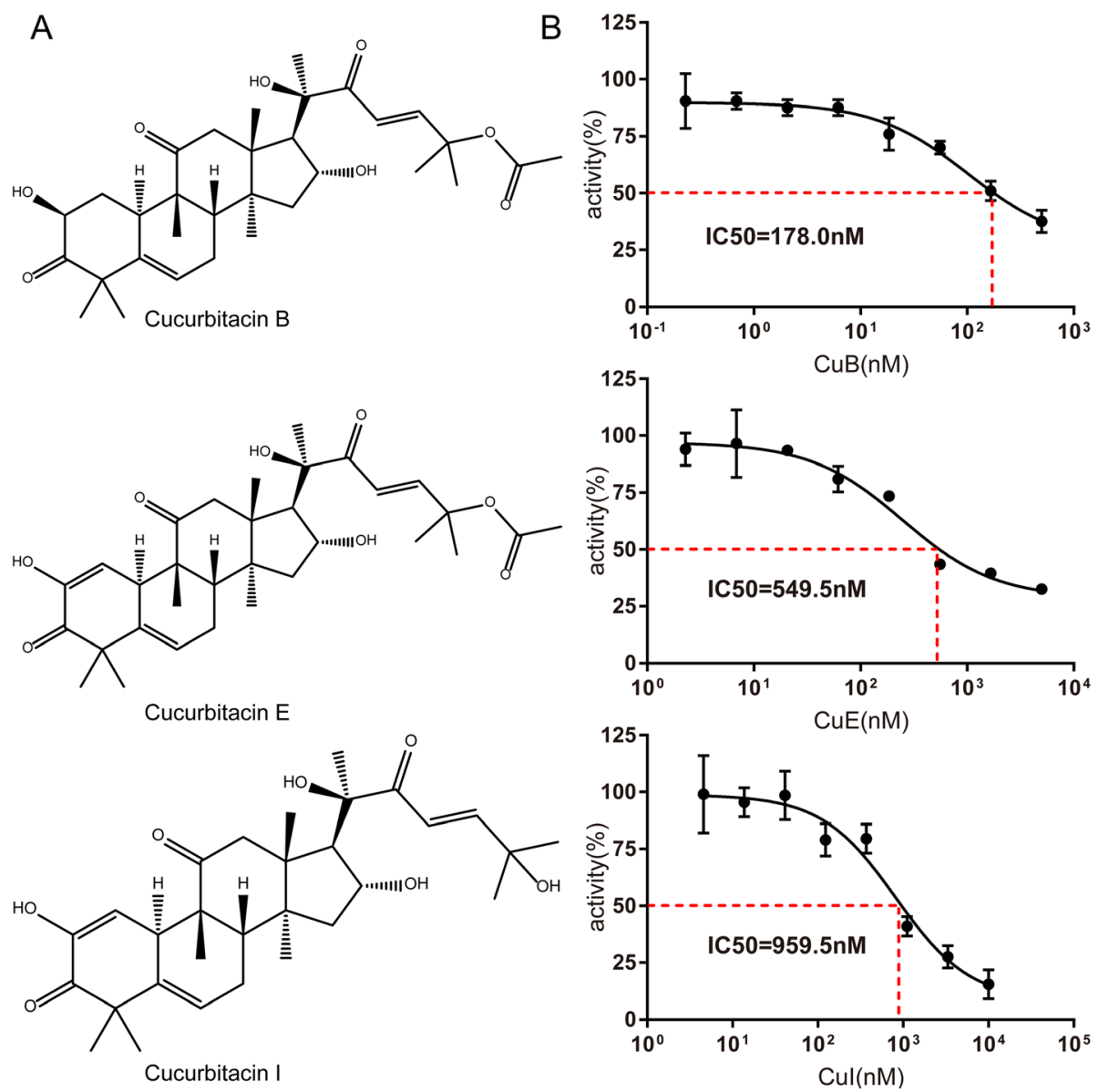

Fig. 1 Cucurbitacins inhibit Tropomyosin receptor kinase A (TrkA) kinase activity at the cellular level. (a) Chemical structure of cucurbitacin B (CuB), CuE, and Cul. (b) TrkA activity in the presence or absence of increasing concentrations of CuB, CuE, and Cul. The results are presented as the percentage of kinase activity relative to the control (dimethyl sulfoxide), and the average of at least duplicate reactions, in which similar results were obtained

could inhibit phosphorylation of Aurora A in human multiple myeloma cells and induce their G2-M arrest and apoptosis [10]. Therefore, $\mathrm{Cu}$ exerts extensive pharmacological activity by regulating multiple cellular pathways, which promoted us to explore more potential targets of $\mathrm{Cu}$.

High-throughput kinase screening provides a powerful platform to support profiling a compound against hundreds of pharmaceutical targets in a single screen [11]. Kinases catalyze the transfer of the $\gamma$-phosphate of ATP to a target substrate. A sequence of such phosphorylation events has been implicated in the modulation of various biological process, including proliferation, survival, apoptosis, metabolism, transcription, and differentiation [12]. Kinase phosphorylation is the basis of cell homeostasis, and aberrant kinase functions have been linked to malignant, inflammatory, and neurodegenerative disorders. Thus, human kinases are a major focal point of basic and drug discovery research. Many effective drugs act by hitting multiple targets rather than individual targets, which may be associated with superior efficacy and toxicity [13]. The kinase target of $\mathrm{Cu}$ has not yet been completely characterized, so a highthroughput kinase screen was carried out. In this study, we used kinase screening approaches to identify tropomyosin receptor kinase $\mathrm{A}(\operatorname{Trk} \mathrm{A})$ as a target of $\mathrm{Cu}$.

\section{Methods}

\section{Reagents, cells and cell culture}

The PC12 cells used in the present study were obtained from the American Type Culture Collection (ATCC, Rockville, MD, USA). The cells were maintained in Dulbecco's modified Eagle's medium (DMEM, Gibco, Gaithersburg, MD, USA) containing 10\% (v/v) fetal bovine serum (FBS) and 5\% horse serum, supplemented with penicillin/streptomycin (all from Gibco). Cus were obtained from Sigma-Aldrich (St. Louis, MO, USA) and were dissolved in dimethyl sulfoxide (DMSO) at a stock concentration of $10 \mathrm{mM}$. The TrkA kinase inhibitor, GW441756, was purchased from Selleck Chemicals 
(Houston, TX, USA). The drugs were dissolved in DMSO to an initial stock concentration of $10 \mathrm{mM}$, and aliquots were made and stored at $-80^{\circ} \mathrm{C}$ until use.

\section{Plant materials}

The Gua Di (Cucumis melo fruit peduncles) were collected from Wulian County $\left(35^{\circ} 45^{\prime} 0.76^{\prime \prime} \mathrm{N}, 119^{\circ} 12^{\prime} 11.01^{\prime \prime}\right.$ E, altitude $272 \mathrm{~m}$ ), Rizhao City, Shandong Province, China. Bitter gourd (Momordica charantia) leaves were collected from Xindu District $\left(30^{\circ} 49^{\prime} 35.10^{\prime \prime} \mathrm{N}, 104^{\circ} 09^{\prime} 19.77^{\prime \prime} \mathrm{E}\right.$, altitude $500 \mathrm{~m}$ ), Chengdu City, Sichuan Province, China in July 2016. Voucher specimens (Voucher number- GD01, BG01) have been deposited in the School of Pharmacy, Chengdu Medical College, Chengdu, China. These plants were identified by Professor Dr. Yuhang chen, Department of Pharmacy, The Chengdu Medical College.

\section{Ethanol extraction}

Gua Di and Bitter gourd leaves were separately pulverized into fine powder using a stainless-steel blender and mixed with ethanol in a 1:10 ratio (w/v). The material was then extracted by boiling in a flask under reflux twice $(1 \mathrm{~h}$ per cycle). Then, the extracts were concentrated under vacuum in a rotary evaporator. The decoction was lyophilized and stored at $4{ }^{\circ} \mathrm{C}$. The lyophilized powder was dissolved in DMSO and filtered through a $0.22 \mu \mathrm{m}$ syringe filter to create a stock solution. GDE and BGLE denote Gua Di and Bitter gourd leaf ethanol extract, respectively. GDE and BGLE were diluted with culture medium to the final concentration indicated for each experiment.

\section{HPLC analysis}

The HPLC analysis was performed on a Waters 2695 apparatus coupled to a Waters 2996 photodiode array detector, assisted by Waters Empower 2 software. A Kromasil $100-5 \mathrm{C} 18(5 \mu \mathrm{m}, 250 \times 4.6 \mathrm{~mm})$ column was used for analytical HPLC (mobile phase, 70\% methanol, $1 \mathrm{~mL} / \mathrm{min}$ ), with the absorbance detection at $230 \mathrm{~nm}$.

\section{Primary kinase screening}

All assays were carried out by the International Centre for Protein Kinase Profiling (http://www.kinase-screen. mrc.ac.uk/) as described previously [14]. Briefly, all determinations were performed at room temperature and were in a linear range with respect to time and enzyme concentration. Assays were performed for $30 \mathrm{~min}$ using Multidrop Micro reagent dispensers (Thermo Fisher Scientific, San José, CA, USA) in a 96-well format. The concentration of magnesium acetate in the assays was $10 \mathrm{mM}$ and $\left[\gamma^{-33} \mathrm{P}\right]$ ATP $(\sim 800 \mathrm{cpm} / \mathrm{pmol})$ was used at $5 \mu \mathrm{M}$ for protein kinases. The assays were stopped by adding $5 \mu \mathrm{L}$ of $0.5 \mathrm{M}$ (3\%) orthophosphoric acid, and were then harvested onto Whatman ${ }^{\circ}$ P81 UNIFILTER ${ }^{\circ}$ plates with a washing buffer containing $50 \mathrm{mM}$ orthophosphoric acid.

\section{Protein kinase activity assays}

The assays to determine the $50 \%$ inhibitory concentration (IC50) values for each enzyme were performed as described previously [15]. In brief, TrkA $(5-20 \mathrm{mU}$ diluted in $50 \mathrm{mM}$ Tris, pH 7.5, $0.1 \mathrm{mM}$ EGTA, 0.1\% $\beta$ mercaptoethanol, $1 \mathrm{mg} / \mathrm{mL}$ bovine serum albumin was assayed against a substrate peptide (Poly Glu Tyr) in a final volume of $25.5 \mu \mathrm{L}$, containing $50 \mathrm{mM}$ Tris, $\mathrm{pH} 7.5$, $0.1 \mathrm{mM}$ EGTA, $1 \mathrm{mg} / \mathrm{mL}$ substrate peptide, $10 \mathrm{mM}$ magnesium acetate, and $0.02 \mathrm{mM}\left[\gamma^{-}{ }^{33} \mathrm{P}\right]$ ATP $(50-1000$ $\mathrm{cpm} / \mathrm{pmole}$ ), and was incubated for $30 \mathrm{~min}$ at room temperature. The assays were stopped by adding $5 \mu \mathrm{L}$ of $0.5 \mathrm{M}$ orthophosphoric acid, and were then harvested onto plates with a wash buffer of $50 \mathrm{mM}$ orthophosphoric acid. Controls for solvents and background phosphorylation levels were included in all assays, and the non-specific ${ }^{33} \mathrm{P}$ incorporation obtained was subtracted from all values. IC50 values were generated from log dose-response curves using Prism 5 software (GraphPad Software Inc., San Diego, CA, USA).

\section{Docking calculations}

TrkA (PDB code: 4PMM) was obtained from the Protein Data Bank. The molecular structures of all compounds were obtained from PubChem. All water atoms were removed, and hydrogen atoms were added to the structures using the pyMol. The nonbonded interactions were calculated using all atomic parameters of AutoDock based on the AMBER force field, since they best reproduced crystal ligand conformations. Implicit solvation parameters were added from the AutoDock Tools package. Ligand nonpolar hydrogen atoms were marked as atom type ' $\mathrm{X}$ '. Docking grids for carbon, oxygen, and polar and nonpolar hydrogen atoms were calculated by AutoGrid, also part of AutoDock. Grid maps contained points for TrkA and all of the compounds to constrain them within the docking cavity. Docking cavities were detected with AutoDockTools, and the candidate cavity was chosen referencing the inhibitor in the crystal structure, 4PMM. The binding conformation was screened with the Genetic Algorithm with Local Search docking method. The docking results were analyzed using pyMol.

\section{Western blot analysis}

PC12 cells $\left(4 \times 10^{6}\right.$ cells $)$ were serum-starved in DMEM in the presence of $\mathrm{CuB}, \mathrm{CuE}, \mathrm{CuI}, \mathrm{GDE}, \mathrm{BGLE}$, and GW441756 for $8 \mathrm{~h}$. NGF (PeproTech, Rocky Hill, NJ) was added to the culture medium at a concentration of $50 \mathrm{ng} / \mathrm{mL}$. After $5 \mathrm{~min}$, the cells were lysed, and the protein concentration of all cell lysate samples was 
measured using the bicinchoninic acid (BCA) protein assay kit (Thermo Fisher Scientific, San José, CA, USA). The cell lysates with equal amounts of protein were mixed with SDS loading buffer containing 10\% SDS, 50 $\mathrm{mM}$ dithiothreitol, $312 \mathrm{mM}$ Tris- $\mathrm{HCl}(\mathrm{pH}=6.8)$, and $50 \%$ glycerin, boiled for $5 \mathrm{~min}$, and separated by SDSPAGE electrophoresis. The proteins were then transferred to polyvinylidene difluoride membranes (Roche Company, Basel, Switzerland). The membranes were blocked in PBS containing 0.05\% (v/v) Tween ${ }^{\circ} 20$ and $5 \%$ $(\mathrm{w} / \mathrm{v})$ nonfat dry milk at room temperature for $1 \mathrm{~h}$. The primary antibody incubations were performed in TBS with $0.1 \%$ Tween ${ }^{\circ} 20$ (TBST) with $5 \%$ nonfat dry milk overnight. After washing, horseradish peroxidase (HRP)linked secondary antibodies (1:4000 dilution) were added and incubated for $1 \mathrm{~h}$. Subsequently, the membranes were washed and incubated in ECL detection reagent (Millipore, Schwalbach, Germany). The following primary antibodies were used: rabbit anti-Phospho-TrkA 490 (1:2000), anti-Phospho-TrkA 674/675 (1:2000), rabbit anti-Phospho-TrkA 785 (1:2000), rabbit antiPhospho-Akt 473(1:2000), rabbit anti-TrkA (1:2000), rabbit anti-Akt (1:2000) and rabbit anti-GAPDH (1: 4000). All antibodies were purchased from Cell Signaling Technology, Danvers, MA, USA.

\section{Statistical analysis}

GraphPad Prism 5.0 software was used to analyze data statistics. The results are presented as means \pm standard deviations.

\section{Results}

\section{Cucurbitacins suppresses TrkA activity}

In the present study, the kinase selectivity of $\mathrm{CuB}$ was assessed using a radioactivity-based enzymatic assay against a kinase panel (Dundee profiling) [16]. $\mathrm{CuB}$ inhibited the kinase activity of TrkA by greater than $90 \%$ at $500 \mathrm{nM}$ (Table 1), with an IC50 value of $178 \mathrm{nM}$ (Fig. 1b), indicating that $\mathrm{CuB}$ is a potent inhibitor of TrkA. Then, we tested the ability of other Cus, CuE and CuI, to inhibit the activity of TrkA. As expected, both $\mathrm{CuE}$ and $\mathrm{CuI}$ significantly inhibited the phosphorylation of TrkA with an IC50 of 549.5 and 959.5 $\mathrm{nM}$, respectively. These screening data provide the first direct evidence of the potential utility of Cus as novel TrkA inhibitors, However, these studies do not explain how Cus binds to the TrkA kinase. Thus, a computational docking study was performed to elucidate the binding mode of the TrkA kinase and its inhibitors.

\section{Docking studies}

To obtain structural models of Cus as TrkA inhibitors, AutoDock was used to explore their binding to TrkA.
The model was generated using the coordinates of known TrkA crystal structures, particularly those of the complex with known TrkA inhibitor (PDB code 4PMM), without crystal water and coligands. Two reasonable binding modes of Cus in the pocket of TrkA were found (Fig. 2), in which Cus and positive controls (GW441756 and K252a) occupy approximately the same pocket (Cavity Volume $858 \AA 3$ ), but are oriented differently. In both binding modes, the compounds, GW441756 and K252a, were the positive TrkA inhibitors. Our docking studies indicate that K252a interacts with TrkA through H-bonds with residues Met592, Tyr391, and Glu590, whereas no $\mathrm{H}$-bond interactions were observed between GW441756 and TrkA. Similar results were obtained with the Cus and TrkA. As expected, CuB interacts with TrkA through $\mathrm{H}$-bonds with residues Tyr591 and Glu590. CuE interacts with TrkA through H-bonds with residues Tyr591 and Met671. CuI interacts with TrkA through H-bonds with residues Tyr591, Ala542, and Met671. The hydrogen-bonding interactions of the amino acids stabilize the complex. Meanwhile, the binding affinity of the TrkA/ligand complexes was expressed in terms of docking scores. Docking scores of GW441756 and K252a against TrkA were - 140.49 and - 160.51, respectively. Similar results were obtained with the Cus and TrkA. The docking scores of $\mathrm{CuB}, \mathrm{CuE}$, and CuI against TrkA were $-138.11,-153.78$, and -153.70 , respectively. The more negative the docking score, the more favorable the interaction of the complex, so these results provide a theoretical possibility that Cus are potential TrkA inhibitors. This corroborates the kinase panel screening data, but it should be further supported by cellular TrkA pathway assays.

\section{Cucurbitacins inhibits NGF-mediated TrkA pathway in PC12 cells}

In this regard, it is noteworthy that pheochromocytoma 12 (PC12) cells express TrkA receptor that mediates neurite outgrowth and anti-apoptotic signaling by binding to NGF [17]. Hence, PC12 cell culture systems have been used to study the effects of potential inhibitors on the kinase activity of TrkA. The PC12 cells were serumstarved for $6 \mathrm{~h}$ and pretreated with various concentrations of $\mathrm{CuB}, \mathrm{CuE}, \mathrm{CuI}$ before being stimulated with NGF $(50 \mathrm{ng} / \mathrm{mL})$. The results of western blot analysis clearly show that NGF stimulated TrkA phosphorylation on several tyrosine sites, including tyrosine 490, 674/ 675, and 785 (Fig. 3). This response was inhibited when the cells were pretreated with $10-100 \mu \mathrm{M} \mathrm{CuB}$ and $\mathrm{CuE}$. Furthermore, $\mathrm{CuI}$ was more effective than $\mathrm{CuB}$ and $\mathrm{CuE}$ were in inhibiting TrkA phosphorylation, with $10 \mu \mathrm{M} \mathrm{CuI}$ significantly inhibiting TrkA phosphorylation (Fig. 3a). The inhibitory activity on TrkA phosphorylation induced by $\mathrm{CuE}$ seems to be the lowest, and $\mathrm{CuE}$ even induced the 
Table 1 Effect of cucurbitacin B (CuB) on protein kinase activities in a high-throughput screening

\begin{tabular}{|c|c|c|c|c|c|c|c|}
\hline Kinase & $\begin{array}{l}\text { Remaining } \\
\text { activity(\%) }\end{array}$ & Kinase & $\begin{array}{l}\text { Remaining } \\
\text { activity(\%) }\end{array}$ & Kinase & $\begin{array}{l}\text { Remaining } \\
\text { activity(\%) }\end{array}$ & & $\begin{array}{l}\text { Remaining } \\
\text { activity(\%) }\end{array}$ \\
\hline TrkA & $5.2 \pm 0.8$ & BRSK2 & $74.9 \pm 1.6$ & HIPK3 & $91.3 \pm 4.0$ & PHK & $106.4 \pm 8.4$ \\
\hline CAMK1 & $12.9 \pm 3.7$ & ERK5 & $75.1 \pm 10.6$ & PKD1 & $91.6 \pm 11.2$ & IRAK4 & $107.5 \pm 9.8$ \\
\hline AMPK & $22.6 \pm 1.5$ & MKK1 & $75.7 \pm 3.8$ & TIE2 & $92.2 \pm 2.5$ & p38d MAPK & $108.1 \pm 21.4$ \\
\hline Aurora B & $23.2 \pm 2.8$ & $\mathrm{IKKb}$ & $76.5 \pm 18.1$ & BRSK1 & $93.3 \pm 2.0$ & CK2 & $108.3 \pm 32.0$ \\
\hline YES1 & $29.8 \pm 3.7$ & MAPK-K3 & $77.2 \pm 1.4$ & MNK1 & $94.2 \pm 15.4$ & ERK2 & $108.5 \pm 14.9$ \\
\hline TSSK1 & $33.8 \pm 5.0$ & MLK3 & $77.4 \pm 13.5$ & ROCK 2 & $94.8 \pm 3.4$ & DAPK1 & $108.5 \pm 18.5$ \\
\hline PIM3 & $40.6 \pm 5.2$ & SmMLCK & $77.4 \pm 10.9$ & PKBa & $94.9 \pm 14.1$ & PRK2 & $108.7 \pm 14.7$ \\
\hline PAK4 & $42.3 \pm 4.6$ & CK1Y2 & $77.6 \pm 21.0$ & JNK2 & $95.3 \pm 1.0$ & PKA & $109.2 \pm 15.3$ \\
\hline PKCa & $43.9 \pm 2.4$ & CLK2 & $78.5 \pm 5.5$ & TAK1 & $95.5 \pm 18.3$ & DDR2 & $109.4 \pm 7.8$ \\
\hline HER4 & $46.2 \pm 15.1$ & MELK & $79.5 \pm 14.7$ & STK33 & $96.3 \pm 7.0$ & MST2 & $109.6 \pm 30.2$ \\
\hline DYRK1A & $47.4 \pm 4.9$ & CSK & $80.9 \pm 5.6$ & WNK1 & $97.0 \pm 1.4$ & OSR1 & $109.6 \pm 2.0$ \\
\hline PKCY & $50.1 \pm 8.0$ & GCK & $81.2 \pm 11.9$ & CDK9 & $97.4 \pm 14.2$ & JAK2 & $109.7 \pm 8.0$ \\
\hline PAK2 & $53.1 \pm 5.5$ & EPH-B3 & $81.8 \pm 2.8$ & ULK2 & $98.6 \pm 4.1$ & MINK1 & $112.6 \pm 15.4$ \\
\hline ERK8 & $54.3 \pm 2.7$ & MSK1 & $82.7 \pm 6.8$ & PDGFRA & $99.2 \pm 8.5$ & NEK6 & $113.0 \pm 24.7$ \\
\hline EF2K & $55.1 \pm 1.2$ & PINK & $83.2 \pm 9.7$ & CK1ס & $99.3 \pm 28.6$ & MEKK1 & $114.2 \pm 13.1$ \\
\hline CHK2 & $56.2 \pm 1.9$ & MAP4K3 & $83.9 \pm 22.2$ & IRAK1 & $99.5 \pm 2.4$ & NEK2a & $114.4 \pm 11.3$ \\
\hline MKK2 & $57.0 \pm 6.9$ & $\mathbb{I R}$ & $84.1 \pm 5.7$ & SRPK1 & $99.6 \pm 13.2$ & TGFBR1 & $116.0 \pm 26.8$ \\
\hline NUAK1 & $58.5 \pm 11.6$ & SIK2 & $84.5 \pm 12.6$ & RSK1 & $99.7 \pm 5.0$ & MNK2 & $116.8 \pm 19.1$ \\
\hline PIM1 & $59.5 \pm 13.7$ & PDK1 & $84.6 \pm 9.2$ & TTBK2 & $100.3 \pm 11.8$ & EPH-A2 & $118.8 \pm 15.6$ \\
\hline SYK & $61.3 \pm 5.8$ & SGK1 & $84.9 \pm 4.2$ & ASK1 & $100.5 \pm 17.0$ & SIK3 & $120.9 \pm 26.3$ \\
\hline PAK5 & $62.3 \pm 6.9$ & MAPK-K2 & $86.6 \pm 9.9$ & ERK1 & $101.6 \pm 4.4$ & TESK1 & $121.0 \pm 11.0$ \\
\hline FGF-R1 & $62.3 \pm 7.4$ & TAO1 & $87.0 \pm 1.7$ & ULK1 & $102.0 \pm 11.2$ & BTK & $122.0 \pm 45.3$ \\
\hline VEG-FR & $65.4 \pm 4.3$ & LKB1 & $87.0 \pm 11.2$ & IGF-1R & $102.4 \pm 3.9$ & MKK6 & $125.9 \pm 6.4$ \\
\hline HIPK2 & $65.6 \pm 1.6$ & p38a MAPK & $87.3 \pm 13.8$ & TTBK1 & $102.5 \pm 14.3$ & PAK6 & $127.3 \pm 2.3$ \\
\hline CDK2 & $66.7 \pm 3.6$ & Src & $87.4 \pm 14.8$ & TLK1 & $102.5 \pm 25.8$ & EPH-A4 & $127.5 \pm 5.0$ \\
\hline DYRK2 & $67.5 \pm 5.0$ & MARK1 & $87.6 \pm 11.4$ & MST4 & $102.8 \pm 6.4$ & MPSK1 & $127.9 \pm 43.9$ \\
\hline MARK4 & $70.5 \pm 1.5$ & RIPK2 & $88.1 \pm 22.1$ & BRK & $103.0 \pm 24.0$ & IRR & $131.0 \pm 37.3$ \\
\hline IKKe & $71.2 \pm 12.5$ & PIM2 & $88.3 \pm 3.9$ & ZAP70 & $104.0 \pm 8.2$ & p38g MAPK & $131.3 \pm 22.8$ \\
\hline DYRK3 & $71.6 \pm 2.7$ & MAP4K5 & $88.9 \pm 15.4$ & MST3 & $104.3 \pm 10.8$ & p38b MAPK & $139.7 \pm 0.8$ \\
\hline TTK & $71.9 \pm 2.6$ & MLK1 & $89.1 \pm 3.6$ & RSK2 & $105.0 \pm 11.4$ & PKCz & $145.1 \pm 17.1$ \\
\hline JNK3 & $72.2 \pm 13.0$ & JNK1 & $89.8 \pm 7.8$ & CAMKKb & $105.7 \pm 2.0$ & Lck & $157.4 \pm 11.1$ \\
\hline PRAK & $72.8 \pm 0.1$ & PLK1 & $89.8 \pm 13.2$ & MARK2 & $105.8 \pm 0.2$ & EPH-B4 & $160.4 \pm 40.7$ \\
\hline GSK3b & $73.2 \pm 8.2$ & PKBb & $90.0 \pm 11.3$ & MARK3 & $105.9 \pm 5.5$ & EPH-B1 & $190.1 \pm 4.6$ \\
\hline S6K1 & $73.7 \pm 27.0$ & EIF2AK3 & $90.7 \pm 9.1$ & $A B L$ & $106.2 \pm 23.9$ & CHK1 & $190.6 \pm 4.0$ \\
\hline EPH-B2 & $74.3 \pm 6.4$ & TBK1 & $91.2 \pm 12.5$ & HIPK1 & $106.4 \pm 27.1$ & & \\
\hline
\end{tabular}

Results presented indicate the remaining activity of each kinase (average of two duplicate determinations \pm standard deviation) in the presence of $500 \mathrm{nM}$ of CuB. Inhibition of tropomyosin receptor kinase A (TrkA) by CuB is indicated in red font

increased phosphorylation at TrkA490 site of TrkA at the higher concentration may due to CuE's complicated biological mechanism (Fig. 3b). In PC12 cells, TrkA has been shown to promote sustained activation of the PI3K/ Akt pathway, leading to increased cell survival [17]. Thus, upstream TrkA blockade is expected to inhibit downstream Akt phosphorylation. Indeed, we verified experimentally that Cus inhibit downstream Akt phosphorylation (Fig. 3).
This provides further evidence that Cus are TrkA inhibitors.

\section{GDE and BGLE inhibits NGF-mediated TrkA pathway in PC12 cells}

'Gua Di' (in Chinese), a traditional Chinese herb prescribed for liver disorders by native Chinese physicians, is rich in $\mathrm{CuB}$ and $\mathrm{CuE}$ [18]. It is noteworthy that Gua 


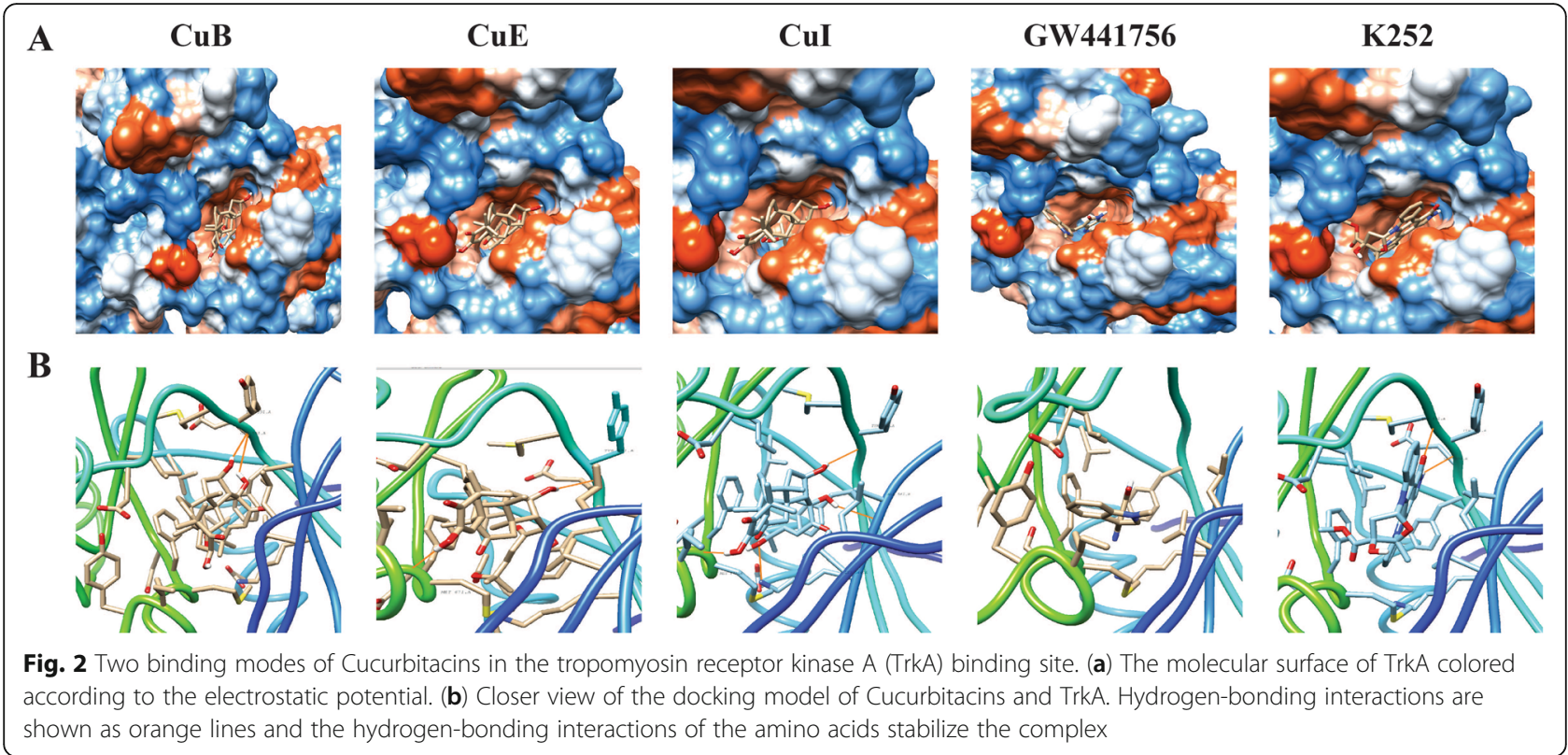

Di has also been used as a folk remedy to treat pruritus. TrkA aberrant activation plays important roles in pruritus and psoriatic plaque formation, and TrkA kinase inhibitor has been shown to be an effective and novel agent to treat patients with pruritus due to psoriasis [19]. To understand the mechanisms of anti-pruritus induced by Gua Di, the inhibitory effect of GDE on TrkA phosphorylation was evaluated. First, HPLC analysis of the original sample (Fig. 4a) indicates that the GDE contained $\mathrm{CuI}$ (retention time: $8.90 \mathrm{~min}$ ), $\mathrm{CuB}$ (retention time: $11.50 \mathrm{~min}$ ), and $\mathrm{CuE}$ (retention time: $12.80 \mathrm{~min}$ ).
The fractions corresponding to peaks 1, 2, and 3 contained $\mathrm{CuI}, \mathrm{CuB}$, and $\mathrm{CuE}$, respectively, as confirmed via HPLC analysis using the $\mathrm{Cu}$ standards. Second, GDE caused a dose-dependent decrease in TrkA phosphorylation in starved PC12 cells; this effect was consequently associated with decreased phosphorylation of TrkA at Y490, Y674, Y675, and Y785. Interestingly, folk wisdom believes that bitter gourd ( $M$. charantia L) leaf helps to prevent or counteract pruritus, and that the plants of the genera Momordica contain a special group of Cus [1]. Thus, we wanted to investigate if BGLE possessed the

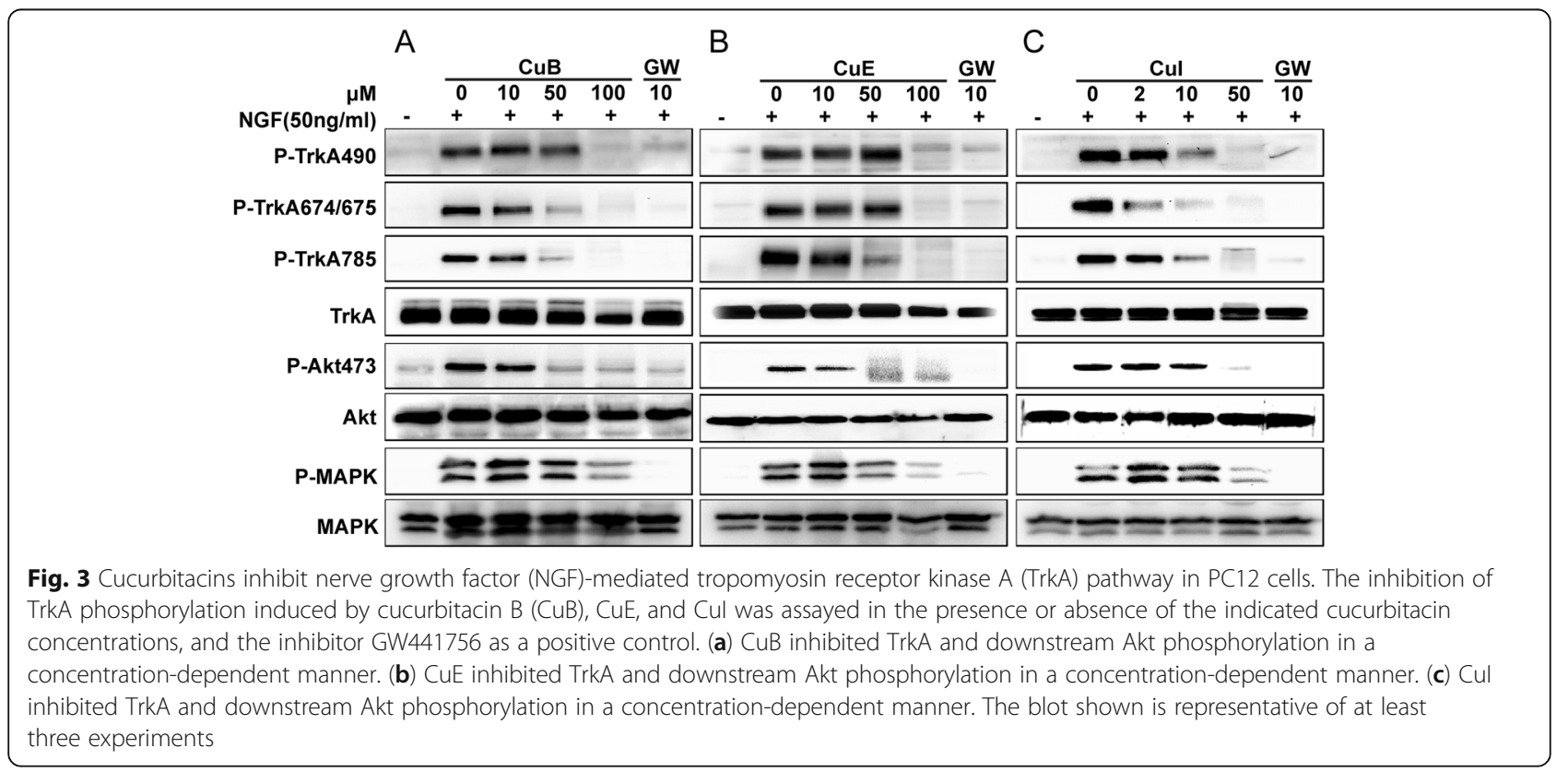


A
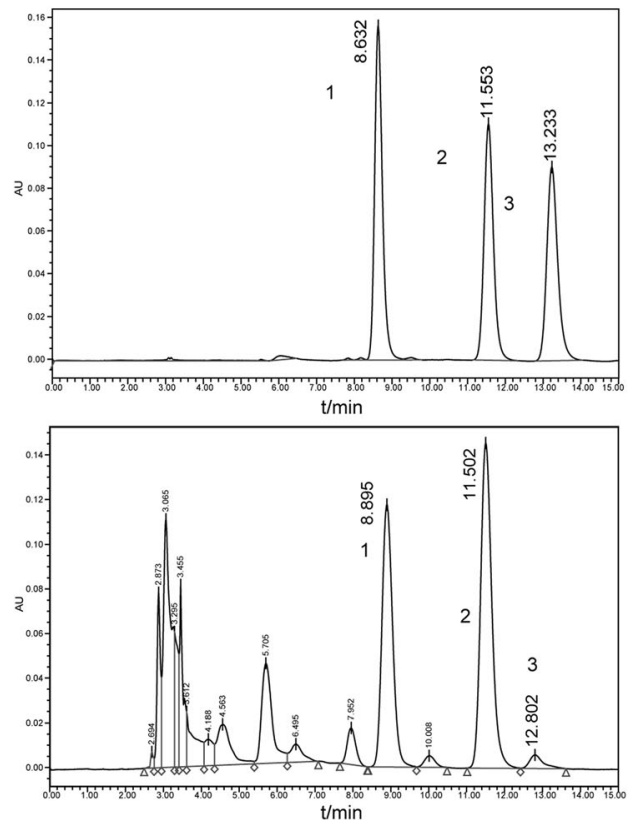

B
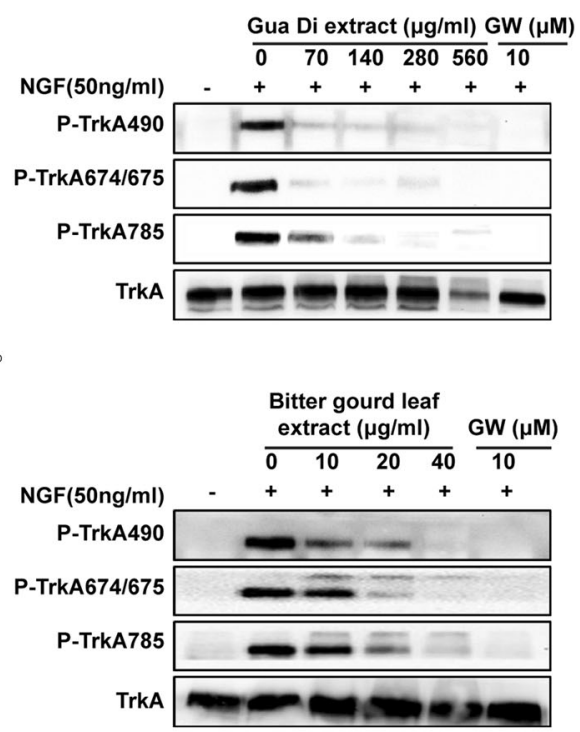

Fig. 4 Gua Di extract (GDE) and Bitter gourd leaf extract (BGLE) inhibit nerve growth factor (NGF)-mediated tropomyosin receptor kinase A (TrkA) pathway in PC12 cells. (a) HPLC chromatogram of three standard compounds Cul (1), CuB (2) and CuE (3) respectively (upper panel); HPLC chromatograms of extracts from GDE detected at $230 \mathrm{~nm}$ (lower panel). Key to peak identities: cucurbitacin I (Cul) (1); CuB (2);CUE (3); (b) GDE (upper panel) and BGLE (lower panel) inhibited TrkA a phosphorylation in a concentration-dependent manner as shown by western blot

ability to inhibit TrkA activity, similar to that of the $\mathrm{Cu}$ derivatives. Our results show that BGLE does indeed inhibit TrkA phosphorylation from PC12 cells significantly in a dose-dependent manner (Fig. 4b).

\section{Discussion}

Aberrant kinase function or regulation can contribute to the rise of many diseases [20]. While protein kinases have become therapy targets, only a small fraction of protein kinases are targeted by validated inhibitors [21]. High-throughput screening technology has become a key tool to screen several compounds against kinases quickly and effectively [21, 22]. In this study, we used kinase screening approaches to identify kinase targets of $\mathrm{CuB}$. The strongest inhibitory activity was found against TrkA. TrkA, the surface transmembrane receptor tyrosine kinase for the neurotrophin, nerve growth factor (NGF), plays an important role in the pathogenesis of psoriasis and associated pruritus [23, 24]. Supporting this possibility is recent clinical evidence that TrkA kinase inhibition significantly reduced pruritus in patients with psoriasis [19]. Thus, TrkA, which plays a key role in the development and maintenance of cutaneous innervation, has emerged as a new therapeutic target for developing anti-pruritus treatments. To our knowledge, the present study is the first identification of Cus as TrkA kinase inhibitors that can be used to inhibit classical NGF/TrkA activity in cells. These data contribute to the use of $\mathrm{Cu}$ derivatives as lead compounds for the design and development of new agents against diseases with abnormal TrkA activation.

Unlike other protein kinase inhibitors currently in use, there have been relatively few reports of TrkA inhibitors. Most of these molecules share a similar structure with staurosporine. For example, both CEP-701 and K252a are TrkA inhibitors that are structurally related to staurosporine. Thus, characterization of novel classes of potent and specific TrkA inhibitors is still a challenge. Due to their important therapeutic promise, significant efforts to identify novel TrkA inhibitors have been made in recent years. The natural product, wrightiadione, was discovered as a new template for the development of TrkA inhibitors. The wrightiadione derivative, $2 \mathrm{~h}$, showed a potent inhibitory activity (IC50 $=6.6 \mu \mathrm{M})$ toward TrkA at the molecular level [25]. In the present study, we describe $\mathrm{Cu}$ as a novel, cell-permeable inhibitor class of TrkA, which specifically inhibits TrkA with an IC50 value of $178-959.5 \mathrm{nM}$. CuI is the most potent, inhibiting NGF-mediated TrkA phosphorylation at the cellular level at $10 \mu \mathrm{M}$. Due to their potency as TrkA inhibitors, Cus provide an attractive platform to design derivatives with enhanced inhibitory activity toward TrkA.

The discovery of artemisinin came from an intensive search for plant natural products representing the wisdom of Chinese medicine [26, 27]. The application of cucurbitacin against chronic hepatitis is another 
successful example of Chinese medicine's influence on innovative drug discovery. The Ben Cao Gang Mu, published in 1596, describes the characteristics and applications of all the medicines listed, and it records the usage of Gua Di for treating icterus..In addition, Gua Di was described by Wang Tao in his treatise "Wai Tai Mi Yao" (Chinese Tang Dynasty). This prescription is well known for the treatment of skin-related diseases such as psoriasis, pruritus, and dermatitis. The fact that Cus inhibit TrkA provides a possible molecular mechanism to explain this effect.

There is evidence that the NGF-TrkA pathway plays a key role in the pathogenesis of psoriasis and associated pruritus. Human mast cells express functional TrkA, are a source of NGF, and NGF-induced histamine secretion from mast cells induces itch or pruritus [28, 29]. Keratinocytes also synthesize and secret NGF, and keratinocytes derived from psoriatic plaques synthesize higher levels of NGF compared to that of normal subjects [30, 31]. Abnormal NGF production induces pruritus through the sensitization of the peripheral sensory nerve terminals in the skin, the majority of which express TrkA. Thus, inhibition of the NGF-TrkA pathway is an effective strategy for treating pruritus.

\section{Conclusion}

In conclusion, Cus block the phosphorylation of TrkA on several tyrosine sites, including Tyr490, Tyr674/675, and Tyr785, and inhibit downstream Akt and MAPK phosphorylation in response to NGF in PC12 cell model systems. Furthermore, traditional Chinese medicinal plants (Tian Gua Di and bitter gourd leaf) containing $\mathrm{Cu}$ extracts were shown to inhibit the phosphorylation of TrkA and Akt. These data reveal mechanisms, at least partly, of the anti-pruritus bioactivity of Cus. Considering the development of this TrkA inhibitor, together with the recent discovery about the important role of TrkA as a therapeutic target, $\mathrm{Cu}$ could act as a precursor for the development of improved TrkA kinase inhibitors with enhanced activity, which might one day contribute to the treatment of pruritus due to psoriasis.

\section{Abbreviations \\ Cu: Cucurbitacin; NGF: Nerve growth factor; TrkA: Tropomyosin receptor kinase $\mathrm{A}$}

\section{Acknowledgments}

We thank the staff at the National Centre for Protein Kinase Profiling (www. kinase-screen.mrc.ac.uk) for undertaking the kinase screening.

\section{Author's contributions}

$J L$ and TY conceived and designed the experiments; YLZ, HX, and XMZ performed the experiments; YZ, LML, GJX and MHL analyzed the data; TY wrote the paper. All authors read and approved the final manuscript.

\section{Funding}

The authors declare that this work was supported by the Scientific Research Fund of the National Natural Science Foundation of China (NO. 81201668),
Chengdu Science and Technology Bureau (2015-HM01-00506-SF), Scientific Research Fund of the Sichuan Provincial Education Department (NO. 17CZ0011, 17ZA0109), Scientific Research Fund of Chengdu Medical College (NO. CYCG15-01), Scientific Research Fund of CMA - L'OREAL China Skin/Hair (S2016-25), National Undergraduates Innovating Experimentation Project (201613705009, 201613705070, 201613705092).

\section{Availability of data and materials}

The datasets and study materials will be available from the corresponding author on request.

Ethics approval and consent to participate

Not applicable.

\section{Consent for publication}

Not applicable.

\section{Competing interests}

The authors declare that they have no competing interests.

\section{Author details}

${ }^{1}$ School of Pharmacy, Chengdu Medical College, No.783, Xindu Avenue, Xindu District, Chengdu 610500, Chengdu Sichuan Province, China. ${ }^{2}$ School of Laboratory Medicine, Chengdu Medical College, Chengdu, China. ${ }^{3}$ Center for Scientific Research, Chengdu Medical College, No.783, Xindu Avenue, Xindu District, Chengdu 610500, Sichuan Province, China.

Received: 16 July 2018 Accepted: 3 October 2019

Published online: 06 November 2019

\section{References}

1. Kaushik U, Aeri V, Mir SR. Cucurbitacins - an insight into medicinal leads from nature. Pharmacogn Rev. 2015;9(17):12-8.

2. Sikander M, Hafeez BB, Malik S, Alsayari A, Halaweish FT, Yallapu MM, Chauhan SC, Jaggi M. Cucurbitacin D exhibits potent anti-cancer activity in cervical cancer. Sci Rep. 2016;6:36594.

3. Hung CM, Chang CC, Lin CW, Chen CC, Hsu YC. GADD45gamma induces G2/M arrest in human pharynx and nasopharyngeal carcinoma cells by cucurbitacin E. Sci Rep. 2014:4:6454.

4. Rios JL, Andujar I, Escandell JM, Giner RM, Recio MC. Cucurbitacins as inducers of cell death and a rich source of potential anticancer compounds. Curr Pharm Des. 2012;18(12):1663-76.

5. Dong Y, Lu B, Zhang X, Zhang J, Lai L, Li D, Wu Y, Song Y, Luo J, Pang X, et al. Cucurbitacin $E$, a tetracyclic triterpenes compound from Chinese medicine, inhibits tumor angiogenesis through VEGFR2-mediated Jak2STAT3 signaling pathway. Carcinogenesis. 2010:31(12):2097-104.

6. Zheng Q, Liu Y, Liu W, Ma F, Zhou Y, Chen M, Chang J, Wang Y, Yang G, He G. Cucurbitacin B inhibits growth and induces apoptosis through the JAK2/ STAT3 and MAPK pathways in SHSY5Y human neuroblastoma cells. Mol Med Rep. 2014;10(1):89-94

7. Nakashima S, Matsuda H, Kurume A, Oda Y, Nakamura S, Yamashita M, Yoshikawa M. Cucurbitacin E as a new inhibitor of cofilin phosphorylation in human leukemia U937 cells. Bioorg Med Chem Lett. 2010;20(9):2994-7.

8. Zhang $Y$, Ouyang $D, X u L$, Ji $Y$, Zha Q, Cai J, He X. Cucurbitacin B induces rapid depletion of the $\mathrm{G}$-actin pool through reactive oxygen speciesdependent actin aggregation in melanoma cells. Acta Biochim Biophys Sin Shanghai. 2011;43(7):556-67.

9. Zhu JS, Ouyang DY, Shi ZJ, Xu LH, Zhang YT, He XH. Cucurbitacin B induces cell cycle arrest, apoptosis and autophagy associated with $\mathrm{G}$ actin reduction and persistent activation of cofilin in Jurkat cells. Pharmacology. 2012;89(56):348-6.

10. Yang $T$, Liu J, Yang $M$, Huang $N$, Zhong $Y$, Zeng $T$, Wei R, Wu Z, Xiao C, Cao $X$, et al. Cucurbitacin B exerts anti-cancer activities in human multiple myeloma cells in vitro and in vivo by modulating multiple cellular pathways. Oncotarget. 2017;8(4):5800-13.

11. Goldstein DM, Gray NS, Zarrinkar PP. High-throughput kinase profiling as a platform for drug discovery. Nat Rev Drug Discov. 2008;7(5):391-7.

12. Munoz L. Non-kinase targets of protein kinase inhibitors. Nat Rev Drug Discov. 2017.

13. Hopkins AL. Network pharmacology: the next paradigm in drug discovery Nat Chem Biol. 2008;4(11):682-90. 
14. Pearce LR, Alton GR, Richter DT, Kath JC, Lingardo L, Chapman J, Hwang C, Alessi DR. Characterization of PF-4708671, a novel and highly specific inhibitor of p70 ribosomal S6 kinase (S6K1). Biochem J. 2010;431(2):245-55.

15. Tyler RK, Shpiro N, Marquez R, Eyers PA. VX-680 inhibits Aurora a and Aurora B kinase activity in human cells. Cell Cycle. 2007;6(22):2846-54.

16. Vogt J, Traynor R, Sapkota GP. The specificities of small molecule inhibitors of the TGFss and BMP pathways. Cell Signal. 2011;23(11):1831-42.

17. Shimoke K, Sasaya H, Ikeuchi T. Analysis of the role of nerve growth factor in promoting cell survival during endoplasmic reticulum stress in PC12 cells. Methods Enzymol. 2011;490:53-70.

18. Chen X, Bao J, Guo J, Ding Q, Lu J, Huang M, Wang Y. Biological activities and potential molecular targets of cucurbitacins: a focus on cancer. AntiCancer Drugs. 2012;23(8):777-87.

19. Roblin D, Yosipovitch G, Boyce B, Robinson J, Sandy J, Mainero V, Wickramasinghe R, Anand U, Anand P: Topical TrkA kinase inhibitor CT327 is an effective, novel therapy for the treatment of pruritus due to psoriasis: results from in vitro and tissue experimental studies, and efficacy and safety of CT327 in a phase 2b, Randomised, Double-blind, Vehicle-controlled Clinical Trial in Patients with Psoriasis. Acta dermato-venereologica 2015

20. Liu M, Zhao G, Cao S, Zhang Y, Li X, Lin X. Development of certain protein kinase inhibitors with the components from traditional Chinese medicine. Front Pharmacol. 2016;7:523.

21. Duong-Ly KC, Peterson JR. A high-throughput radiometric kinase assay. Methods Mol Biol. 2016;1360:87-95.

22. Ma H, Horiuchi KY. Chemical microarray: a new tool for drug screening and discovery. Drug Discov Today. 2006;11(13-14):661-8.

23. Schulte-Herbruggen $\mathrm{O}$, Folster-Holst $R$, von Elstermann $M$, Augustin $M$, Hellweg R. Clinical relevance of nerve growth factor serum levels in patients with atopic dermatitis and psoriasis. Int Arch Allergy Immunol. 2007;144(3):211-6.

24. Yamaguchi J, Aihara M, Kobayashi Y, Kambara T, Ikezawa Z. Quantitative analysis of nerve growth factor (NGF) in the atopic dermatitis and psoriasis horny layer and effect of treatment on NGF in atopic dermatitis. J Dermatol Sci. 2009;53(1):48-54

25. Jeong Y, Lim SM, Hong S. Discovery of wrightiadione as a novel template for the TrkA kinase inhibitors. Bioorg Med Chem Lett. 2015;25(22):5186-9.

26. Shen B. A new Golden age of natural products drug discovery. Cell. 2015; 163(6):1297-300

27. Tu Y. Artemisinin-a gift from traditional Chinese medicine to the world (Nobel lecture). Angew Chem. 2016;55(35):10210-26.

28. Nilsson G, Forsberg-Nilsson K, Xiang Z, Hallbook F, Nilsson K, Metcalfe DD. Human mast cells express functional TrkA and are a source of nerve growth factor. Eur J Immunol. 1997;27(9):2295-301.

29. Stempelj M, Ferjan I. Signaling pathway in nerve growth factor induced histamine release from rat mast cells. Inflammation research : official journal of the European Histamine Research Society [et al]. 2005;54(8):344-9.

30. Pincelli C, Sevignani C, Manfredini R, Grande A, Fantini F, Bracci-Laudiero L, Aloe L, Ferrari S, Cossarizza A, Giannetti A. Expression and function of nerve growth factor and nerve growth factor receptor on cultured keratinocytes. The Journal of investigative dermatology. 1994;103(1):13-8.

31. Pincelli C. Nerve growth factor and keratinocytes: a role in psoriasis. European journal of dermatology : EJD. 2000;10(2):85-90.

\section{Publisher's Note}

Springer Nature remains neutral with regard to jurisdictional claims in published maps and institutional affiliations.

Ready to submit your research? Choose BMC and benefit from:

- fast, convenient online submission

- thorough peer review by experienced researchers in your field

- rapid publication on acceptance

- support for research data, including large and complex data types

- gold Open Access which fosters wider collaboration and increased citations

- maximum visibility for your research: over $100 \mathrm{M}$ website views per year

At BMC, research is always in progress.

Learn more biomedcentral.com/submissions 